\title{
Case Study for Bank ATM Queuing Model
}

\author{
S. K. Dhar ${ }^{1}$, Tanzina Rahman ${ }^{2}$ \\ ${ }^{1,2}$ (Department of EEE, University of Information Technology \& Sciences(UITS), Bangladesh)
}

\begin{abstract}
Queuing is the common activity of customers or people to avail the desired service, which could be processed or distributed one at a time. Bank ATMs would avoid losing their customers due to a long wait on the line. The bank initially provides one ATM in every branch. But, one ATM would not serve a purpose when customers withdraw to use ATM and try to use other bank ATM. Thus the service time needs to be improved to maintain the customers. This paper shows that the queuing theory used to solve this problem. We obtain the data from a bank ATM in a city. We then derive the arrival rate, service rate, utilization rate, waiting time in the queue and the average number of customers in the queue based on the data using Little's theorem and M/M/I queuing model. The arrival rate at a bank ATM on Sunday during banking time is 1 customer per minute (cpm) while the service rate is $1.50 \mathrm{cpm}$. The average number of customer in the ATM is 2 and the utilization period is 0.70 . We conclude the paper by discussing the benefits of performing queuing analysis to a busy ATM.
\end{abstract}

Keywords: Bank ATM, Little's theorem, M/M/I queuing model, Queue, Waiting lines.

\section{INTRODUCTION}

Queue is a common word that means a waiting line or the act of joining a line. Queuing theory was initially proposed by A.K. Erlang in 1903. It optimizes the number of service facilities and adjusts the times of services [1]. Queuing theory is the study of queue or waiting lines. Some of the analysis that can be derived using queuing theory include the expected waiting time in the queue, the average time in the system, the expected queue length as well as the probability of the system to be in certain states, such as empty or full. This paper uses queuing theory to study the waiting lines in BRAC BANK ATM, at Chittagong city, Bangladesh. The bank provides one ATM in every branch. In ATM, bank customers arrive randomly and the service time is also random. We use Little's theorem and M/M/I queuing model to derive the arrival rate, service rate, utilization rate, waiting time in the queue. On average, 600 customers are served on weekdays (Sunday to Thursday) and 300 customers are served on weekends (Friday \& Saturday) monthly. Generally, on Sundays, there are more customers coming to ATM, during $10 \mathrm{am}$. to $6 \mathrm{pm}$.

\section{QUEUING SYSTEM}

A queuing system can be completely described by

i) the input or arrival pattern (customers);

ii) the service mechanism (service pattern);

iii) the 'queue discipline'; \&

iv) customer's behaviour

The diagrammatic representation of the above components of queuing system is shown in the figure 1:

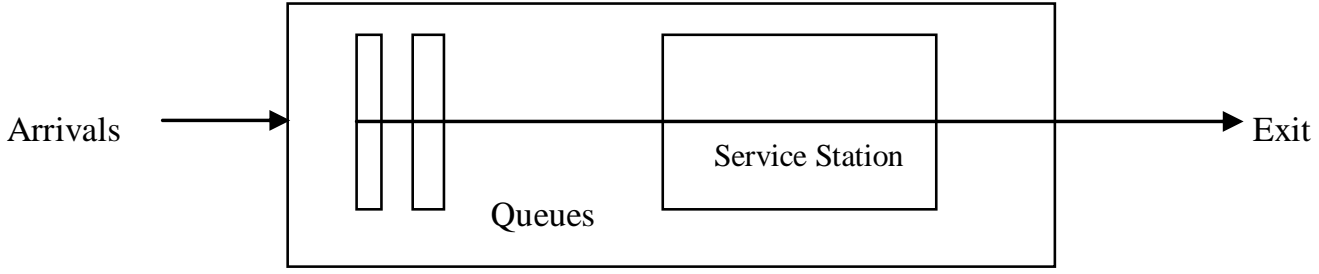

Figure 1: Queuing System

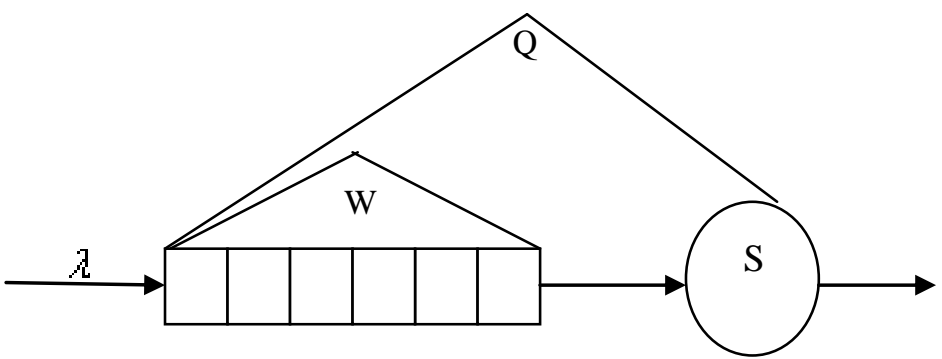

Figure 2: Queuing Model 
A queuing system consists of one or more servers that provide service to arriving customers. Figure 1 shows the characteristics of queuing system [2,3]. The population of customers may be finite (closed systems) or infinite (open systems). The customers arrive to the service center in a random fashion.

Queue represents a certain number of customers waiting for service. The capacity of a queue is either limited or unlimited. Bank is an example of unlimited queue length.

Figure 2 represents a queuing model, where

- $\quad$ Arrival rate $(\lambda)$ - the average rate at which customers arrive.

- $\quad$ Service time (s) - the average time required to service one customer.

- $\quad$ Number waiting $(\mathrm{W})$ - the average number of customers waiting.

- $\quad$ Number in the system $(\mathrm{Q})$ - the total number of customers in the system.

\section{QUEUING THEORY}

In 1908, Copenhagen Telephone Company requested Agner K. Erlang to work on the holding times in a telephone switch. He identified that the number of telephone conversations and telephone holding time fit into Poisson distribution and exponentially distributed. This was the beginning of the study of queuing theory. In this section, we will discuss two common concepts in queuing theory.

\subsection{Little's theorem}

Little's theorem [4] describes the relationship between throughput rate (i.e. arrival \& service rate), cycle time and work in process (i.e. number of customers/jobs in the system). The theorem states that the expected number of customers $(N)$ for a system in steady state can be determined using the following equation:

$$
L=\lambda T
$$

Here, $\lambda$ is the average customer arrival rate and $T$ is the average service time for a customer.

Three fundamental relationships can be derived from Little's theorem [5]:

- $\quad L$ increases if $\lambda$ or $T$ increases

- $\quad \lambda$ increases if $L$ increases or $T$ decreases

- $\quad T$ increases if $L$ increases or $\lambda$ decreases

\subsection{ATM Model (M/M/I queuing model)}

$\mathrm{M} / \mathrm{M} / 1$ queuing model means that the arrival and service time are exponentially distributed (Poisson process). For the analysis of the ATM M/M/1 queuing model, the following variables will be investigated:

- $\lambda$ : The mean customers arrival rate

- $\mu$ : The mean service rate

- $\rho=\frac{\lambda}{\mu}:$ Utilization factor

- Probability of zero customers in the ATM:

$$
P_{0}=1-\rho
$$

- $\quad P_{n}$ : The probability of having $n$ customers in the ATM:

$$
P_{n}=P_{0} \rho^{n}=(1-\rho) \rho^{n}
$$

- $\quad L$ : The average number of customers in the ATM:

$$
L=\frac{\rho}{1-\rho}=\frac{\lambda}{\mu-\lambda}
$$

- $\quad L_{q}$ : The average number of customers in the queue:

$$
L_{q}=L \times \rho=\frac{\rho^{2}}{1-\rho}=\frac{\rho \lambda}{\mu-\lambda}
$$

- $\quad W_{\mathrm{q}}$ : The average waiting time in the queue:

$$
W_{q}=\frac{L_{q}}{\lambda}=\frac{\rho}{\mu-\lambda}
$$

- $W$ : The average time spent in the ATM, including the waiting time:

$$
W=\frac{L}{\lambda}=\frac{1}{\mu-\lambda}
$$




\section{OBSERVATION \& DisCUSSION}

We have collected the one month daily customer data by observation during banking time, as shown in Table -1

Table -1 [Monthly Customer Counts]

\begin{tabular}{|c|c|c|c|c|c|c|c|}
\hline \multirow{2}{*}{$\begin{array}{c}\text { Day } \\
\text { Week }\end{array}$} & Weekend & \multicolumn{5}{|c|}{ Weekdays } & Weekend \\
\cline { 2 - 8 } & Saturday & Sunday & Monday & Tuesday & Wednesday & Thursday & Friday \\
\hline $1^{\text {st }}$ & 70 & 191 & 161 & 120 & 130 & 111 & 95 \\
\hline $2^{\text {nd }}$ & 75 & 154 & 151 & 110 & 93 & 75 & 80 \\
\hline $3^{\text {rd }}$ & 65 & 140 & 135 & 121 & 100 & 128 & 77 \\
\hline $4^{\text {th }}$ & 50 & 115 & 102 & 92 & 85 & 80 & 78 \\
\hline Total & $\mathbf{2 6 0}$ & $\mathbf{6 0 0}$ & $\mathbf{5 4 9}$ & $\mathbf{4 4 3}$ & $\mathbf{4 0 8}$ & $\mathbf{3 9 4}$ & $\mathbf{3 7 0}$ \\
\hline
\end{tabular}

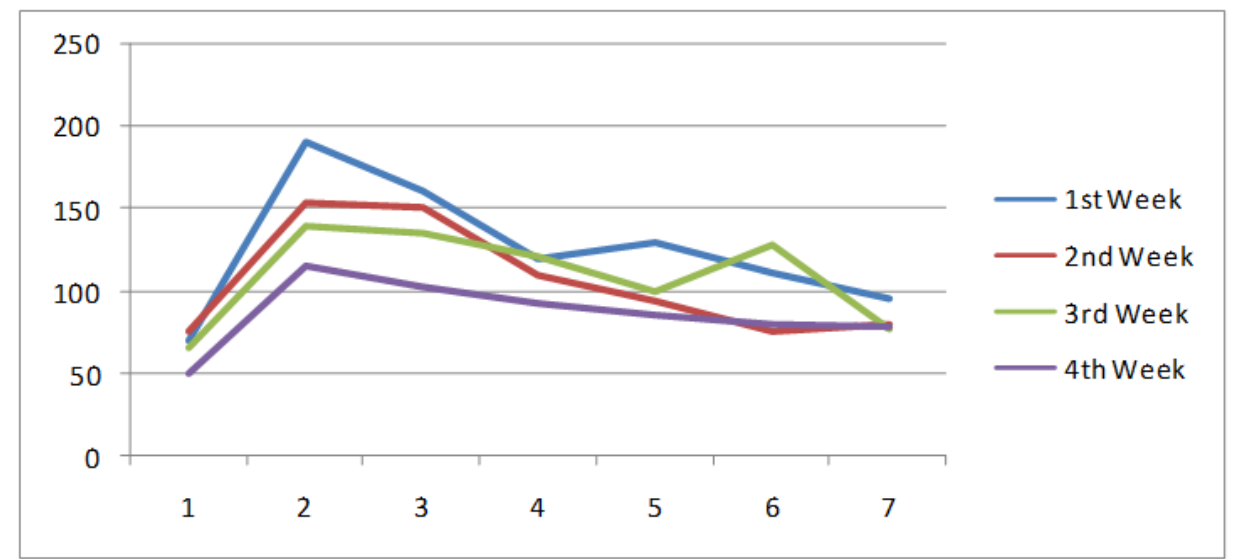

Figure -3 [ One month daily customer counts]

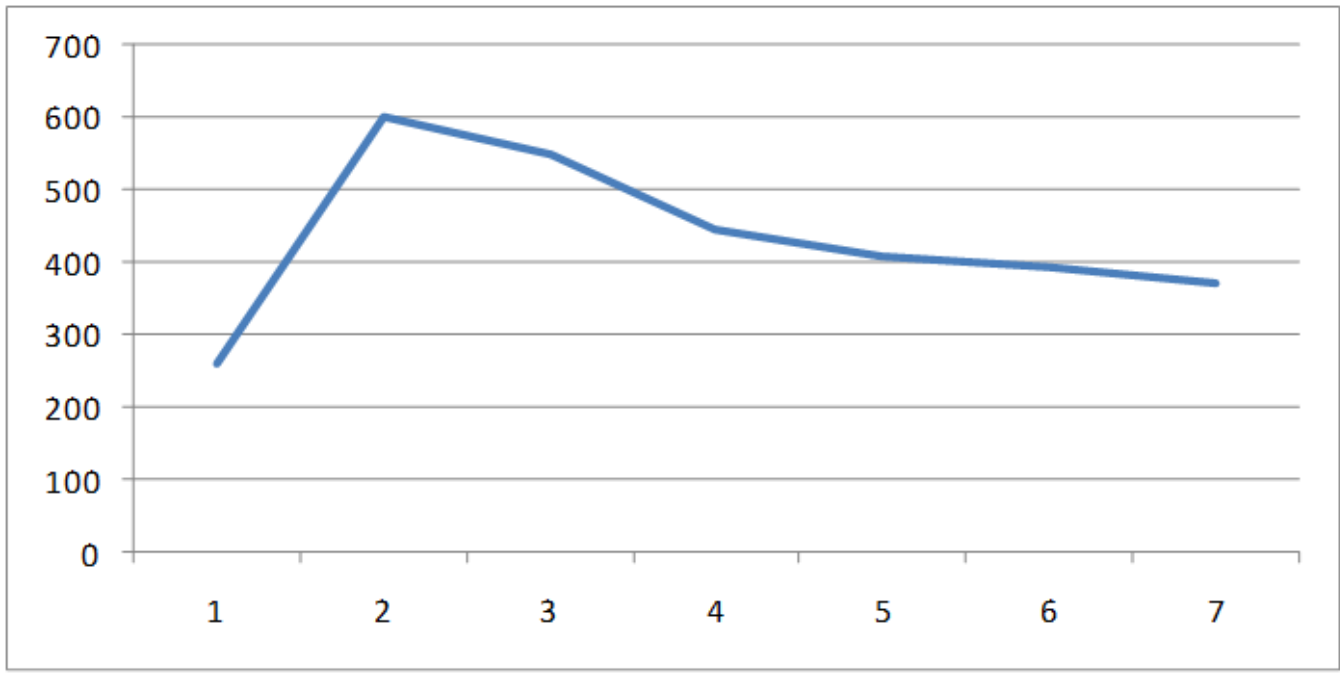

Figure -4 [One month total customer counts]

From the above figure -3 , we observe that, the number of customers on Sundays is double the number of customers on Saturdays during a month. The busiest period for the bank ATM is Sundays and Mondays during banking time $(10 \mathrm{am}$. to $6 \mathrm{pm}$.). Hence, the time period is very important for the research. Also, we observed from the figure -4 that, after Sunday, the number of customers start decreasing slowly as the week progresses. On Saturdays it is least and on Thursdays \& Fridays it stays slightly more than Saturdays. This is because the Fridays and Saturdays will be holiday.

\subsection{Calculation}

We investigate that, after Saturday, during first two days of a week, there are on average 60 people coming to the ATM in an hour time period of banking time. From this we can derive the arrival rate as: 


$$
\lambda=\frac{60}{60}=1 \text { customer } / \text { minute }(\mathrm{cpm}) .
$$

We also found out from observation discussion with the guard that each customer spends 2 minutes on average in the ATM $(W)$, the queue length is around 3 people $(L q)$ on average and the average waiting time is around 2.5 minutes i.e. 150 seconds.

Theoretically, the average waiting time is, $W_{q}=\frac{L_{q}}{\lambda}=\frac{3 \text { customers }}{1 \text { cpm }}=3$ mimutes $=180$ seconds

Here, we can see that, the observed actual waiting time dose not differ by much when it is compared with the theoretical waiting time.

Next, we will calculate the average number of people in the ATM using (1),

$$
L=1 \mathrm{cpm} \times 2 \text { minutes }=2 \text { customers }
$$

Having calculated the average number of customers in the ATM, we can also derive the service rate and the utilization rate using (5) \& (2).

$$
\begin{aligned}
\mu & =\frac{\lambda(1+L)}{L}=\frac{1(1+2)}{2}=1.5 \mathrm{cpm} \\
\text { Hence, } \rho & =\frac{\lambda}{\mu}=\frac{1 \mathrm{cpm}}{1.5 \mathrm{cpm}}=0.70
\end{aligned}
$$

With the very high utilization rate of 0.70 during banking time, the probability of zero customers in the ATM is very small as can be derived using (3),

$$
P_{0}=1-\rho=1-0.70=0.30
$$

The queuing theory provides the formula to calculate the probability of having $n$ customers in the ATM as follows:

$$
P_{n}=(1-\rho) \rho^{n}=(1-0.70)(0.70)^{n}=(0.30)(0.70)^{n}
$$

We assume that impatient customers will start to balk when they see more than 3 people are already queuing for the ATM. We also assume that the maximum queue length that a patient customer can tolerate is 10 people. As the capacity of the ATM is 1 people, we can calculate the probability of 4 people in the system (i.e. in the ATM).

Therefore, the probability of customers going away $=P$ (more than 3 people in the queue) $=P($ more than 4 people in the ATM $)$ is

$$
P_{5-11}=\sum_{m=5}^{11} P_{n}=0.15423=15.42 \%
$$

\subsection{Evaluation}

The utilization is directly proportional with the mean number of customers. It means that the mean number of customers will increase as the utilization increase.

The utilization rate at the ATM is very high at 0.70 . This, however, is only the utilization rate during banking time on Sundays and Mondays. On weekend, the utilization rate is almost half of it. This is because the number of customers on weekends is only half of the number of people on weekdays.

In case the customers waiting time is lower or in other words, we waited for less than 150 seconds, the number of customers that are able to be served per minute will increase. When the service rate is higher, the utilization will be lower, which makes the probability of the customers going away decreases.

\subsection{Benefits}

This research can help bank ATM to increase its QoS (Quality of Service), by anticipating, if there are many customers in the queue. The result of this paper work may become the reference to analyze the current system and improve the next system. Because the bank can now estimate the number of customers waits in the queue and the number of customers going away each day.

By estimating the number of customers coming and going in a day, the bank can set a target that, how many ATMs are required to serve people in the main branch or any other branch of the bank.

\section{CONCLUSION}

This research paper has discussed the application of queuing theory to the Bank ATM. From the result we have obtained that, the rate at which customers arrive in the queuing system is 1 customer per minute and the service rate is 1.50 customers per minute. The probability of buffer flow if there are 3 or more customers in the queue is 10 out of 100 customers. The probability of buffer overflow is the probability that customers will run away, because may be they are impatient to wait in the queue. This theory is also applicable for the bank, if they want to calculate all the data daily and this can be applied to all branch ATM also. The constraints that were 
faced for the completion of this research were the inaccuracy of result since some of the data that we use was just based on assumption or approximation. We hope that this research can contribute to the betterment of a bank in terms of its functioning through ATM.

As our future work, we will develop a simulation model for the bank ATM. By developing a simulation model we will be able to confirm the results of the analytical model that we develop in this paper. By this model, it can mirror the actual operation of the ATM more closely.

\section{REFERENCES}

[1] N. K. Tiwari \& Shishir K. Shandilya, Oprations Research. Third Edition, ISBN 978-81-203-2966-9. Eastern Economy Edition, 2009.

[2] Nita H. Shah, Ravi M. Gor, Hardik Soni, Operations Research. $4^{\text {th }}$ Edition, ISBN 978-81- 203-3128-0. Eastern Economy Edition, 2010 .

[3] K. Sanjay, Bose, “An Introduction to queuing system”, Springer, 2002.

[4] J. D. C. Little, “A Proof for the Queuing Formula: $L=\lambda W$ ", Operations Research, vol. 9(3), 1961, pp. 383-387, doi: $10.2307 / 167570$

[5] M. Laguna and J. Marklund, Business Process Modeling, Simulation and Dsign. ISBN 0-13-091519-X. Pearson Prentice Hall, 2005 . 